\title{
Correspondence and Communications
}

\section{Microsurgical lymphedema treatment: An objective evaluation of the quality of online information}

\author{
Dear Sir,
}

World-wide, almost 300 million people are affected by lymphedema. Patients report severe pain, morbidity, and significant worsening of their quality of life, with meaningful financial burden on both the patient and the healthcare system.

For centuries, physicians have been the main point of reference for patients regarding all sort of medical information. Nowadays, self-informed patients are quite common, thanks to the World Wide Web that offers access to almost 20,000-100,000 health-related websites. ${ }^{1}$ As well, patients suffering from lymphedema seek for information about their condition and treatment options. Despite its evident utility, the quality of online health-related information remains doubtful, as it can be uploaded without any editorial examination, bearing unfiltered statements that are often unscientific, non-exhaustive and unclear. Guidelines and checklists to assess the quality of online health information have been developed. Among these, the expanded EQIP (Ensuring Quality Information for Patients) scale is a checklist that can be used to evaluate the quality of information of any kind of source. ${ }^{2}$

We assessed online health information regarding prepectoral breast reconstruction and body contouring surgery in postbariatric patients, previously. ${ }^{3,4}$ In the present study, we aim to assess the quality of information that patients affected by lymphedema can find, searching for two surgical physiologic procedures: lymphovenous anastomosis (LVA) and vascularized lymph node transfer (VLNT).

We performed the investigation with the keywords "Lymphedema treatment with lymph node transfer" and "Lymphedema treatment with lymphatic venous anastomosis" with the two most commonly used search engines worldwide: Google and Yahoo. The top 50 websites were systematically evaluated, excluding inappropriate documents, duplicates, video, blogs and scientific papers.

The websites included were organized in five groups (practitioners, hospitals, healthcare portals, professional societies and encyclopedias) and we assessed them with the expanded EQIP scale.
The EQIP scale is made up of 36 questions with three sections: content, identification data and structure. A dual choice to answer (Yes or NO) is possible for every item and every answer is worth 0 or 1 point, with a maximum score of 36. Twenty or more points, which is equivalent to the 75 th percentile, determinate a high score website.

Twenty-eight eligible websites that deal with VLNT and 17 with LVA were selected as suitable for the current study. The mean score was 20,3 points for VLNT websites, with 15 websites $(53,6 \%)$ presenting high score. Results were similar for LVA websites, with mean score equal to 20,9 points and 11 websites $(64,7 \%)$ with high score.

Analysing the results (Tables 1 and 2), we could see poor description of benefits and side-effects especially in quantitative terms of both the procedures. There was a deficiency regarding the alert signs that patients may detect and precautions they may apply. No websites included all the appropriate topic themes. Healthcare portals collected the highest average score of 22,9 points. The analysis of the Identification data demonstrated a low rate of bibliography of evidence-based data used: only 6 websites $(21,4 \%)$ about VLNT and 6 (35, 3\%) of LVA presented it.

There are currently no validated guidelines for the treatment of lymphedema. Most patients are initially treated with non-operative interventions such as lymphatic massage and compression. Physiologic reconstruction, such as LVA and VLNT, has been proposed to address early stage lymphedema, as a problem-oriented approach that acts on the pathophysiologic processes in play. ${ }^{5}$ Resectional procedures are limited to more advanced secondary related lymphedema deformity, but they are associated to significant morbidity, permanent disfigurement and recurrence of symptoms. The specific indications for LVA or VLNT remain unclear in the management of lymphedema, and the anticipated benefits from these physiologic appear highly variable.

Direct access to medical information on the internet allows patients to become participative and to present to their healthcare providers with beliefs and expectations regarding their condition and its potential treatment.

Nevertheless, we must question whether this medical information on the web can be considered reliable.

For what concerns the online information regarding LVA and VLNT, the EQIP test we performed showed interesting results, evidencing lack of some relevant topics. For both procedures, the main part of the websites belongs to the Hospital portals group that scored the lowest scores. 
Table 1 EQIP tool results applied to the 17 eligible websites about Lymphedema treatment with lymphatic venous anastomosis (LVA) research on Google ${ }^{\circledR}$ and $Y_{a h o o}{ }^{\circledR}$.

\section{Question}

\section{Content data}

1. Initial definition of which subjects will be covered

2. Coverage of the above-defined subjects

3. Description of the medical problem

4. Definition of the purpose of the medical intervention

5. Description of treatment alternatives (including no treatment)

6. Description of the sequence of the medical procedure

7. Description of qualitative benefits

8. Description of quantitative benefits

9. Description of qualitative risks and side-effects

10. Description of quantitative risks and side-effects

11. Addressing quality of life issues

12. Description of how potential complications will be dealt with

13. Description of precautions that the patient may take

14. Mention of alert signs that the patient may detect

15. Addressing medical intervention cost and insurance issues

16. Specific contact details for hospital services

17. Specific details of other sources of reliable information/support

18. The document covers all relevant issues on the topic

\section{Identification data}

19. Date of issue or revision

20. Logo of the issuing body

21. Name of persons or entities that produced the document

22. Name of persons or entities that financed the document

23. Short bibliography of evidence-based data used in the document

24. The document states if and how patients were involved/consulted in its production

\section{Structure data}

25. Use of everyday language, explains complex words or jargon

26. Use of generic names for all medications or products

27. Use of short sentences

28. The document personally addresses the reader

29. The tone is respectful

30. Information is clear

31. Information is balanced between risks and benefits

32. Information is presented in a logical order

33. The design and layout are satisfactory

34. Figures or graphs are clear and relevant

35. The document has a named space for the reader's notes

36. The document includes a consent form, contrary to recommendations

\begin{tabular}{ll} 
Yes (\%) & No (\%) \\
$17(100 \%)$ & $0(0 \%)$ \\
$17(100 \%)$ & $0(0 \%)$ \\
$17(100 \%)$ & $0(0 \%)$ \\
$17(100 \%)$ & $0(0 \%)$ \\
$16(94,12 \%)$ & $1(05,88 \%)$ \\
$9(52,94 \%)$ & $8(47,06 \%)$ \\
$16(94,12 \%)$ & $1(05,88 \%)$ \\
$1(05,88 \%)$ & $16(94,12 \%)$ \\
$9(52,94 \%)$ & $8(47,06 \%)$ \\
$0(0 \%)$ & $17(100 \%)$ \\
$16(94,12 \%)$ & $1(05,88 \%)$ \\
$1(05,88 \%)$ & $16(94,12 \%)$ \\
$2(11,76 \%)$ & $15(88,24 \%)$ \\
$1(05,88 \%)$ & $16(94,12 \%)$ \\
$4(23,53 \%)$ & $13(76,47 \%)$ \\
$13(76,47 \%)$ & $4(23,53 \%)$ \\
$8(47,06 \%)$ & $9(52,94 \%)$ \\
$0(0 \%)$ & $17(100 \%)$ \\
$16(94,12 \%)$ & $1(05,88 \%)$ \\
$16(94,12 \%)$ & $1(05,88 \%)$ \\
$14(82,35 \%)$ & $3(17,65 \%)$ \\
$1(05,88 \%)$ & $16(94,12 \%)$ \\
$6(35,29 \%)$ & $11(64,71 \%)$ \\
$0(0 \%)$ & $17(100 \%)$ \\
$16(94,12 \%)$ & $1(05,88 \%)$ \\
$17(100 \%)$ & $0(0 \%)$ \\
$17(100 \%)$ & $0(0 \%)$ \\
$17(100 \%)$ & $0(0 \%)$ \\
$17(100 \%)$ & $0(0 \%)$ \\
$17(100 \%)$ & $0(0 \%)$ \\
$2(11,76 \%)$ & $15(88,24 \%)$ \\
$17(100 \%)$ & $0(0 \%)$ \\
$8(47,06 \%)$ & $9(52,94 \%)$ \\
$12(70,59 \%)$ & $5(29,41 \%)$ \\
$0(0 \%)$ & $17(100 \%)$ \\
$0(0 \%)$ & $17(100 \%)$ \\
\hline
\end{tabular}

The prevalence of the Hospitals' group may be explained by the fact that these surgical procedures are frequently performed in hospitals, perhaps more than in private practice. In particular, the quantitative description of benefits, side effects and alert signs along with precaution that patients may take, resulted inadequate in this group. Similarly, identification data section showed a weak bibliography of evidence-based data used.

Patients may not perceive completely the right view on LVA and VLNT. Physiologic reconstruction for lymphedema generally provides modest benefits, and only for a spe- cific subgroup of patients, with no reduction effect on the chronic dermal damage. Moreover, surgical management of lymphedema does not completely eliminate the need for compression therapy, at least for three months after surgery. The available online information generally does not appear to convey the quantitative benefits, or lack thereof, which can result in patients presenting with unrealistic expectations about the potential to address their lymphedema. Surgeons should guide their patients in this quest, warning them on the potential misinformation they might hit in the web. 
Table 2 EQIP tool results applied to the 28 eligible websites about Lymphedema treatment with lymph node transfer (VLNT) research on Google ${ }^{\circledR}$ and Yahoo ${ }^{\circledR}$.

\begin{tabular}{|c|c|c|}
\hline Question & Yes (\%) & No (\%) \\
\hline \multicolumn{3}{|l|}{ Content data } \\
\hline 1. Initial definition of which subjects will be covered & $28(100 \%)$ & $0(0 \%)$ \\
\hline 2. Coverage of the above-defined subjects & $28(100 \%)$ & $0(0 \%)$ \\
\hline 3. Description of the medical problem & $28(100 \%)$ & $0(0 \%)$ \\
\hline 4. Definition of the purpose of the medical intervention & $28(100 \%)$ & $0(0 \%)$ \\
\hline 5. Description of treatment alternatives (including no treatment) & $22(78.57 \%)$ & $6(21.43 \%)$ \\
\hline 6. Description of the sequence of the medical procedure & $10(35.71 \%)$ & $18(64.29 \%)$ \\
\hline 7. Description of qualitative benefits & 25 (89.29\%) & $3(10.71 \%)$ \\
\hline 8. Description of quantitative benefits & $0(0 \%)$ & $28(100 \%)$ \\
\hline 9. Description of qualitative risks and side-effects & $12(42.86 \%)$ & $16(57.14 \%)$ \\
\hline 10. Description of quantitative risks and side-effects & $1(03.57 \%)$ & $27(96.43 \%)$ \\
\hline 11. Addressing quality of life issues & $25(89.29 \%)$ & $3(10.71 \%)$ \\
\hline 12. Description of how potential complications will be dealt with & $3(10.71 \%)$ & $25(89.29 \%)$ \\
\hline 13. Description of precautions that the patient may take & $2(7.14 \%)$ & $26(92.86 \%)$ \\
\hline 14. Mention of alert signs that the patient may detect & $1(03.57 \%)$ & $27(96.43 \%)$ \\
\hline 15. Addressing medical intervention cost and insurance issues & $13(46.43 \%)$ & $15(53.57 \%)$ \\
\hline 16. Specific contact details for hospital services & 27 (96.43\%) & $1(03.57 \%)$ \\
\hline 17. Specific details of other sources of reliable information/support & $9(32.14 \%)$ & $19(67.86 \%)$ \\
\hline 18. The document covers all relevant issues on the topic & $0(0 \%)$ & $28(100 \%)$ \\
\hline \multicolumn{3}{|l|}{ Identification data } \\
\hline 19. Date of issue or revision & $25(89.29 \%)$ & $3(10.71 \%)$ \\
\hline 20. Logo of the issuing body & $23(82.14 \%)$ & $5(17.86 \%)$ \\
\hline 21. Name of persons or entities that produced the document & $16(57.14 \%)$ & $12(42.86 \%)$ \\
\hline 22. Name of persons or entities that financed the document & $1(03.57 \%)$ & $27(96.43 \%)$ \\
\hline 23. Short bibliography of evidence-based data used in the document & $6(21.43 \%)$ & $22(78.57 \%)$ \\
\hline 24. The document states if and how patients were involved/consulted in its production & $0(0 \%)$ & $28(100 \%)$ \\
\hline \multicolumn{3}{|l|}{ Structure data } \\
\hline 25. Use of everyday language, explains complex words or jargon & $27(96.43 \%)$ & $1(03.57 \%)$ \\
\hline 26. Use of generic names for all medications or products & $28(100 \%)$ & $0(0 \%)$ \\
\hline 27. Use of short sentences & $28(100 \%)$ & $0(0 \%)$ \\
\hline 28. The document personally addresses the reader & $28(100 \%)$ & $0(0 \%)$ \\
\hline 29. The tone is respectful & (C) & $0(0 \%)$ \\
\hline 30. Information is clear & $28(100 \%)$ & $0(0 \%)$ \\
\hline 31. Information is balanced between risks and benefits & $4(14.29 \%)$ & $24(85.71 \%)$ \\
\hline 32. Information is presented in a logical order & $28(100 \%)$ & $0(0 \%)$ \\
\hline 33. The design and layout are satisfactory & $18(64.29 \%)$ & $10(35.71 \%)$ \\
\hline 34. Figures or graphs are clear and relevant & $15(53.57 \%)$ & $13(46.43 \%)$ \\
\hline 35. The document has a named space for the reader's notes & $3(10.71 \%)$ & $25(89.29 \%)$ \\
\hline 36. The document includes a consent form, contrary to recommendations & $0(0 \%)$ & $28(100 \%)$ \\
\hline
\end{tabular}

\section{Funding statement}

None.

\section{Ethic statement}

None.

\section{Declaration of Competing Interest}

None.

\section{Acknowledgments}

None.

\section{References}

1. Wald HS, Dube CE, Anthony DC. Untangling the Web-the impact of Internet use on health care and the physician-patient relationship. Patient Educ Couns 2007;68:218-24.

2. Charvet-Berard Al, Chopard P, Perneger TV. Measuring quality of patient information documents with an expanded EQIP scale. Patient Educ Couns 2008;70:407-11.

3. Marcasciano M, Frattaroli J, Mori FLR, et al. The new trend of pre-pectoral breast reconstruction: an objective evaluation of the quality of online information for patients undergoing breast reconstruction. Aesthetic Plast Surg 2019;43:593-9. 
4. Lo Torto F, Marcasciano M, Frattaroli JM, et al. Quality assessment of online information on body contouring surgery in postbariatric patient. Aesthetic Plast Surg 2020;44:839-46.

5. Ciudad P, Manrique OJ, Bustos SS, et al. Comparisons in long-term clinical outcomes among patients with upper or lower extremity lymphedema treated with diverse vascularized lymph node transfer. Microsurgery 2020;40:130-6.

Federico Lo Torto, Juste Kaciulyte, Francesco LR Mori, Jacopo M Frattaroli

Department of Surgery "P.Valdoni", Unit of Plastic and Reconstructive Surgery, Sapienza University of Rome, Policlinico Umberto I, via Ettore Fieramosca, 200, Roma 00159, Italy

E-mail address: federicolotorto@gmail.com

Marco Marcasciano

Department of Oncologic and Reconstructive Breast Surgery, "Breast Unit Integrata di Livorno, Cecina, Piombino, Elba, Azienda USL Toscana nord ovest", Italy

Donato Casella

Azienda Ospedaliera Universitaria Senese, UOC Chirurgia Oncologica della Mammella, Siena, Italy

Emanuele Cigna, Luigi Losco

Dipartimento di Ricerca Traslazionale e delle Nuove Tecnologie in Medicina e Chirurgia, Università degli Studi di Pisa, Pisa, Italy
Oscar J Manrique

Department of Plastic and Reconstructive Surgery, Mayo Clinic, Rochester, MN, USA

Fabio Nicoli

Northumbria Healthcare NHS Foundation Trust, Newcastle upon Tyne, UK

Pedro Ciudad

Department of Plastic and Burn Surgery, Arzobispo Loayza National Hospital, Lima, Peru

Diego Ribuffo

Department of Surgery "P.Valdoni”, Unit of Plastic and Reconstructive Surgery, Sapienza University of Rome, Policlinico Umberto I, via Ettore Fieramosca, 200, Roma 00159, Italy

(C) 2020 British Association of Plastic, Reconstructive and Aesthetic Surgeons. Published by Elsevier Ltd. All rights reserved.

https://doi.org/10.1016/j.bjps.2020.11.033 